\title{
Article \\ Pericardial Effusion on MRI in Autosomal Dominant Polycystic Kidney Disease
}

\author{
Jin Liu ${ }^{1,2}$, Kana Fujikura ${ }^{3}\left(\mathbb{D}\right.$, Hreedi Dev ${ }^{1} \mathbb{D}$, Sadjad Riyahi ${ }^{1}$, Jon Blumenfeld ${ }^{4,5}$, Jiwon Kim ${ }^{4}$, Hanna Rennert ${ }^{6}$ \\ and Martin R. Prince $1,7, *$ (D)
}

check for

updates

Citation: Liu, J.; Fujikura, K.; Dev, H.; Riyahi, S.; Blumenfeld, J.; Kim, J.;

Rennert, H.; Prince, M.R. Pericardial Effusion on MRI in Autosomal

Dominant Polycystic Kidney Disease. J. Clin. Med. 2022, 11, 1127. https:// doi.org/10.3390/jcm11041127

Academic Editors:

Emmanuel Andrès and

Jonathan Townend

Received: 10 December 2021

Accepted: 18 February 2022

Published: 21 February 2022

Publisher's Note: MDPI stays neutral with regard to jurisdictional claims in published maps and institutional affiliations.

Copyright: () 2022 by the authors. Licensee MDPI, Basel, Switzerland. This article is an open access article distributed under the terms and conditions of the Creative Commons Attribution (CC BY) license (https:// creativecommons.org/licenses/by/ $4.0 /)$.
1 Department of Radiology, Weill Cornell Medicine, New York, NY 10065, USA; jil4018@med.cornell.edu (J.L.); hrd2001@med.cornell.edu (H.D.); sar4002@med.cornell.edu (S.R.)

2 Department of Geriatrics, The Second Affiliated Hospital of Nanjing Medical University, Nanjing 210011, China

3 Department of Cardiology, Saint Francis Hospital, New York, NY 11576, USA; kana.fujikura@chsli.org

4 Department of Medicine, Weill Cornell Medicine, New York, NY 10065, USA; jdblume@nyp.org (J.B.); jik9027@med.cornell.edu (J.K.)

5 The Rogosin Institute, New York, NY 10065, USA

6 Department of Pathology, Weill Cornell Medicine, New York, NY 10065, USA; har2006@med.cornell.edu

7 Columbia College of Physicians and Surgeons, New York, NY 10027, USA

* Correspondence: map2008@med.cornell.edu

\begin{abstract}
Autosomal dominant polycystic kidney disease (ADPKD) has been associated with cardiac abnormalities including mitral valve prolapse and aneurysmal dilatation of the aortic root. Herein, we investigated the potential association of pericardial effusion with ADPKD. Subjects with ADPKD $(n=117)$ and control subjects without ADPKD matched for age, gender and renal function $(n=117)$ undergoing MRI including ECG-gated cine MRI of the aorta and heart were evaluated for pericardial effusion independently by three observers measuring the maximum pericardial effusion thickness in diastole using electronic calipers. Pericardial effusion thickness was larger in ADPKD subjects compared to matched controls (Mann-Whitney $p=0.001$ ) with pericardial effusion thickness $>5 \mathrm{~mm}$ observed in 24 of 117 (21\%) ADPKD subjects compared to 4 of $117(3 \%)$ controls $(p=0.00006)$. Pericardial effusion thickness in ADPKD was associated with female gender patients (1.2 mm greater than in males, $p=0.03)$ and pleural effusion thickness $(p<0.001)$ in multivariate analyses. No subjects exhibited symptoms related to pericardial effusion or required pericardiocentesis. In conclusion, pericardial effusion appears to be more prevalent in ADPKD compared to controls. Although in this retrospective cross-sectional study we did not identify clinical significance, future investigations of pericardial effusion in ADPKD subjects may help to more fully understand its role in this disease.
\end{abstract}

Keywords: ADPKD; pericardial effusion; MRI; echocardiography; pleural effusion

\section{Introduction}

Autosomal dominant polycystic kidney disease (ADPKD) is the most common hereditary cause of chronic kidney disease [1-3] and affects the cardiovascular system with hypertension, intracranial (berry) aneurysms, dolichoectasia of the carotid arteries, mitral valve prolapse and aneurysmal dilatation of the aortic root and proximal aorta [4-8]. We have incidentally noted pericardial effusions in ADPKD subjects while evaluating magnetic resonance imaging (MRI) of research subjects in the Rogosin Institute PKD Repository. In this study, we determined the prevalence of pericardial effusion in ADPKD subjects on MRI compared to a control population without ADPKD matched for age, gender and kidney function. 


\section{Materials and Methods}

This HIPAA-compliant study was approved by the local Institutional Review Board at Weill Cornell Medicine. All subjects in the Rogosin Institute PKD Repository signed informed consent.

\subsection{Study Design and Populations}

This is a retrospective cross-sectional study of existing data and images acquired from October 2018 to December 2021. Inclusion criteria were (1) diagnosis of ADPKD based upon Pei criteria $[9,10]$ and (2) MRI including ECG-gated cine steady-state free precession (SSFP) of the aorta and heart. Exclusion criteria were (1) absence of ECG-gated cardiac images, (2) laboratory data not available within 12 months of the MRI, (3) dialysis or kidney transplantation, (4) surgery within 3 months of MRI and (5) active malignancy (Figure 1).

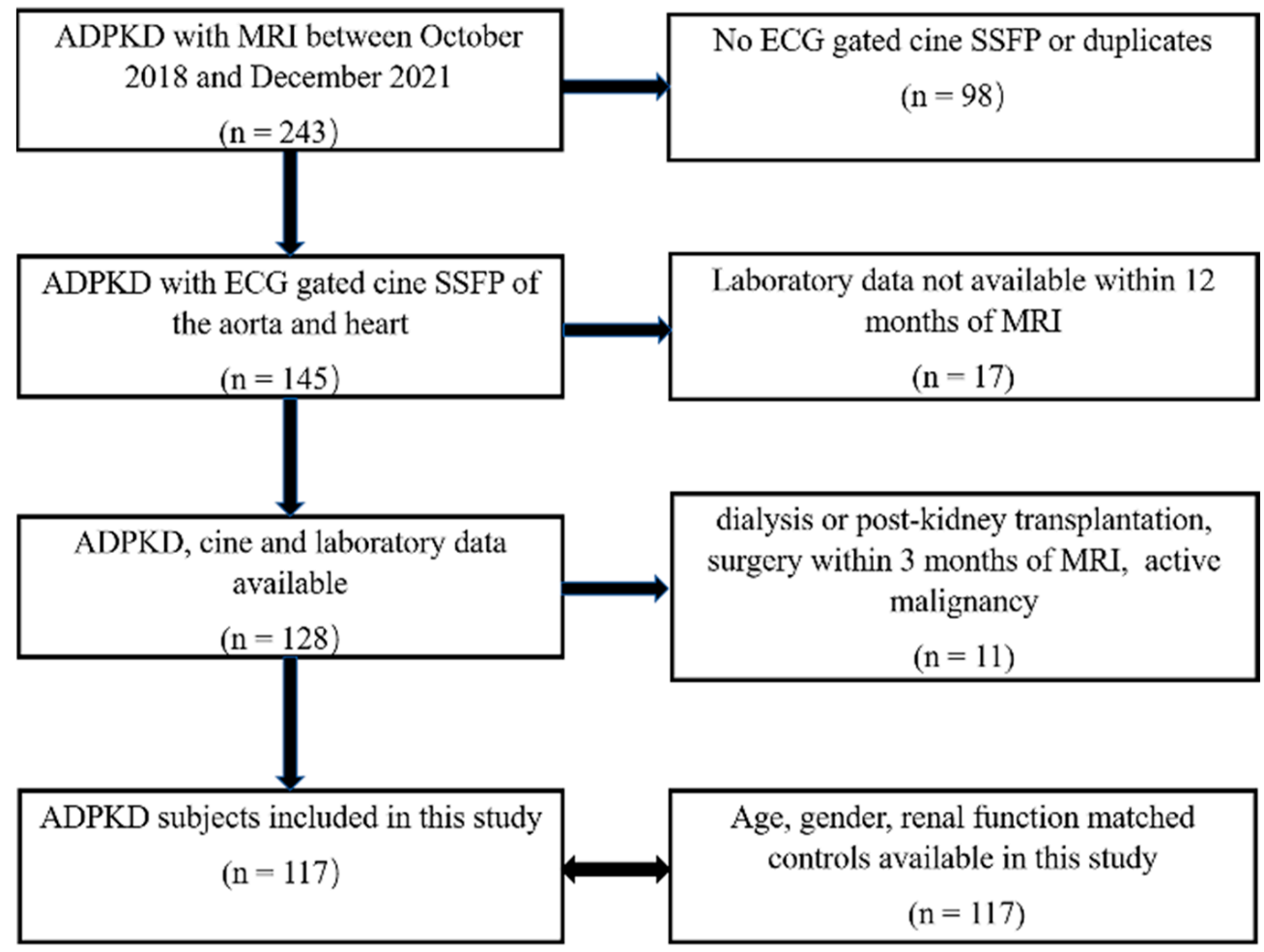

Figure 1. Study flow chart.

Age, gender and estimated glomerular filtration rate (eGFR)-matched control subjects without ADPKD undergoing cardiac MRI were identified from electronic medical records, including the picture archiving computer system (PACS). Additionally, subjects were excluded if they had risk factors for pericardial effusion such as pericarditis, ejection fraction less than $50 \%$, active malignancy or surgery within 3 months of MRI, none of which were seen in the ADPKD group. Subjects with hypothyroidism were also excluded from the control population. Race, when available, was also matched.

\subsection{Data Extraction}

Demographic and laboratory data were extracted from electronic medical records for the date closest to the date of MRI. Data on kidney, liver and spleen volumes and cisterna chyli diameter were extracted from the MRI reports prepared prospectively at the time of imaging. The PKD gene mutation was obtained from the Rogosin Institute PKD Repository. Serum creatinine, blood urea nitrogen (BUN), thyroid-stimulating hormone (TSH), alanine transaminase (ALT), aspartate transaminase (AST) and albumin tests were performed within 1 year of the protocol MRI. eGFR was calculated by the CKD-EPI equation [11]. Data on troponin, copeptin and natriuretic peptides were not available. Where 
available, data on fluid intake were obtained from the electronic medical records, grouping patients into $<3 \mathrm{~L} /$ day or "increased", $\geq 3 \mathrm{~L} /$ day of fluid intake. Data on use of diuretics, tolvaptan and other medications, history of rheumatological disease as well as indications for cardiac MRI in control subjects were obtained from the electronic medical record. Data on echocardiography were extracted from tests performed within 1 year of MRI.

\subsection{Image Acquisition}

All MRI exams were obtained on a $1.5 \mathrm{~T}$ using a body array coil (Signa HDXT, GE Healthcare, Waukesha, WI, USA, or Magnetom Aera, Siemens Healthineers, Erlangen, Germany) using the parameters shown in Table 1. Pulse sequences included, coronal and axial T2-weighted single-shot fast spin echo (SSFSE), 3D spoiled gradient-recalled echo T1-weighted images with fat suppression or Dixon fat-water separation, diffusion weighted imaging (DWI) and sagittal oblique (in the plane of aortic arch) ECG-gated SSFP of the heart and aorta. The cine images were acquired in a single breath hold per slice, reconstructing the R-to-R interval into 20 temporal phases.

Table 1. MRI imaging parameters.

\begin{tabular}{cccccc}
\hline & Coronal SSFSE $^{\mathbf{1}}$ & Axial SSFSE & Axial 3D LAVA $^{2}$ & Axial DWI $^{\mathbf{3}^{2}}$ & $\begin{array}{c}\text { Sagittal SSFP }^{4} \\
\text { Aorta and Heart }^{2}\end{array}$ \\
\hline Field of view & $40-48$ & $30-42$ & $30-42$ & $30-42$ & $30-42$ \\
Matrix & $320 \times 256$ & $320 \times 208$ & $288 \times 192$ & $160 \times 108$ & $240 \times 153$ \\
Slice thickness & $5 \mathrm{~mm}$ & $5 \mathrm{~mm}$ & $3 \mathrm{~mm}$ & $5 \mathrm{~mm}$ & $7 \mathrm{~mm}$ \\
$\mathrm{TR}^{5} / \mathrm{TE}^{6} /$ flip & $1200 / 91 / 130$ & $1000 / 95 / 130$ & $3.98 / 1.24 / 9$ & $7500 / 90 / 90$ & $58 / 1.2 / 39$ \\
\hline
\end{tabular}

${ }^{1}$ SSFSE, single-shot fast-spin echocardiography; ${ }^{2}$ LAVA, liver imaging with volume acceleration; ${ }^{3} \mathrm{DWI}$, diffusionweighted imaging; ${ }^{4} \mathrm{SSFP}$, steady-state free precession; ${ }^{5} \mathrm{TR}$, repetition time; ${ }^{6} \mathrm{TE}$, echocardiography time.

Transthoracic echocardiography was performed using commercial equipment standardized for routine clinical practice. Parasternal long axis, parasternal short axis, apical 4-chamber, apical 2-chamber, apical 3-chamber, subcostal, and suprasternal notch views were acquired in accordance with consensus guidelines [12].

\subsection{Image Analysis}

Sagittal oblique ECG-gated SSFP MRI images were analyzed by 3 independent, experienced observers (JL, KF, MRP) blinded to all subject information (Figure 2A). Pericardial effusions were quantified by measuring the largest dimension of fluid signal in the pericardial space visible during end diastole. One observer (JL) measured subjects three times in order to assess intraobserver variation. For subjects for whom echocardiography data was available, the thickness of pericardial fluid was measured where it appeared largest during end diastole, as shown in Figure 2B. 


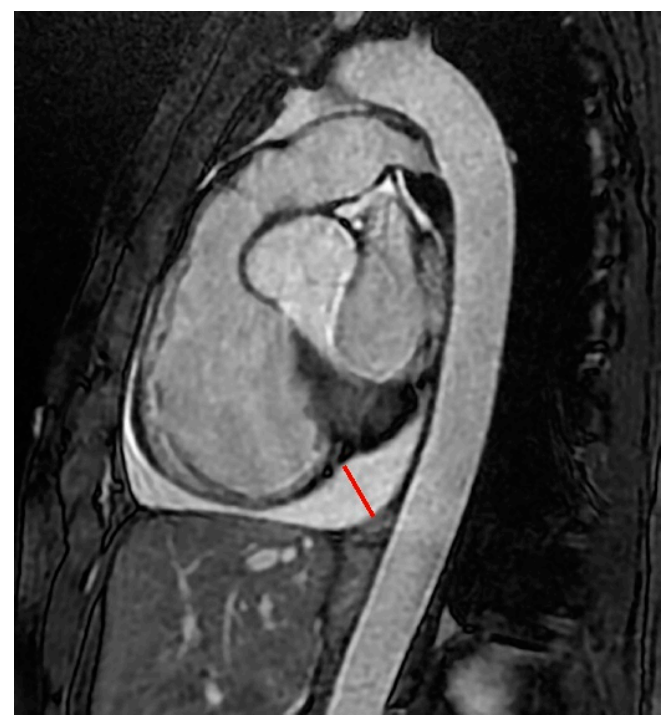

(A)

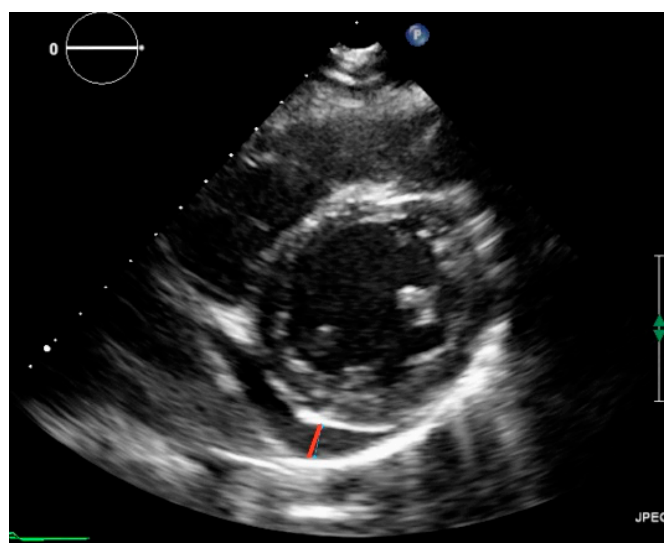

(B)

Figure 2. Example of pericardial effusion measurement in ADPKD subjects on (A) MRI and (B) echocardiography. The measurement of pericardial effusion thickness at end diastole is indicated by the red lines.

\subsection{Statistical Analysis}

All statistical tests were 2-tailed, and a $p$-value $<0.05$ was considered statistically significant. Continuous variables were assessed for normality using the Shapiro-Wilk test and summarized as either mean \pm standard deviation (SD) for normally distributed variables or median (interquartile range) for variables without normal distribution. Frequency and percentage were calculated for categorical variables.

For two-group matched continuous, normally distributed variables, $t$-test was used. The Mann-Whitney test was used for variables that were not normally distributed to assess the statistical significance at $p$-value $<0.05$. For categorical variables, chi-squared test was used to assess statistical significance. The interobserver and intraobserver agreement for measuring pericardial effusion size on MRI was assessed by interclass correlation coefficient (ICC).

Bivariate analysis was used to predict the correlation between the pericardial effusion thickness and the continuous parameters including age, body mass index (BMI), systolic blood pressure, liver volume, kidney volume, spleen volume, cisterna chyli diameter, AST, ALT, albumin, BUN, TSH and eGFR. A multivariate linear regression model was used to predict the mixed effect of the variables found to be significant at $p<0.05$ level upon bivariate analysis while also including age and gender $($ male $=0$, female $=1)$.

\section{Results}

\subsection{Study Population and Characteristics}

ADPKD and matched (age, gender, eGFR) control subjects $(n=117)$ were analyzed in this study (Figure 1, Table 2). Data on race were available for matching in 86 of 117 (74\%) ADPKD subjects. Demographic and laboratory data of this study are shown in Table 2. Although there was a significant difference in serum albumin between ADPKD and control groups, this difference was minor, and all values were within normal ranges. For the control population, the primary indications for cardiac MRI are listed in Supplemental Table S1. The medications used to control blood pressure in control and ADPKD subjects are listed in Supplemental Table S2. The causes of renal insufficiency in the 44 control subjects with eGFR $<60 \mathrm{~mL} / \mathrm{min} / 1.73 \mathrm{~m}^{2}$ are listed in Supplemental Table S3. Echocardiography within 1 year of MRI was available in 46 (39\%) of the 117 ADPKD subjects. 
Table 2. Demographic data in ADPKD subjects with matched controls ${ }^{1}$.

\begin{tabular}{|c|c|c|c|}
\hline Characteristic & $\begin{array}{l}\text { ADPKD Subjects } \\
\quad n=117\end{array}$ & $\begin{array}{l}\text { Matched Controls } \\
\quad n=117\end{array}$ & $p$ Value \\
\hline Age & $47.3 \pm 13$ & $47.8 \pm 13$ & 0.74 \\
\hline Male: female & 50: 67 & 50: 67 & 1 \\
\hline $\begin{array}{l}\text { White: Black: Asian: mixed: Native } \\
\text { American: unknown }\end{array}$ & 76: 10: 8: 6: 1: 16 & 85: 11: 5: 6: 1: 9 & 0.55 \\
\hline Body Mass Index $\left(\mathrm{kg} / \mathrm{m}^{2}\right)$ & $25(23-29)$ & $26(23-30)$ & 0.62 \\
\hline Systolic Blood Pressure (mmHg) & $127(120-136)$ & $125(112-137)$ & 0.16 \\
\hline Diuretic use & $24(21 \%)$ & $26(22 \%)$ & 0.75 \\
\hline Rheumatological disease & $4(3 \%)$ & $8(7 \%)$ & 0.24 \\
\hline Pericardial effusion $(\mathrm{mm})$ mean $\pm \mathrm{SD}$ & $2.9 \pm 3.3$ & $1.6 \pm 1.5$ & 0.001 \\
\hline Pericardial effusion $>5 \mathrm{~mm}$ & $24(21 \%)$ & $4(3 \%)$ & 0.00006 \\
\hline $\begin{array}{l}\text { Estimated Glomerular Filtration Rate } \\
\qquad\left(\mathrm{mL} / \mathrm{min} / 1.73 \mathrm{~m}^{2}\right)\end{array}$ & $67(46-93)$ & $69(52-94)$ & 0.44 \\
\hline Blood Urea Nitrogen (mg/dL) & $19(15-26)$ & $20(15-27)$ & 0.99 \\
\hline Albumin (g/dL) & $4.1(4-4.3)$ & $4(3.7-4.3)$ & 0.004 \\
\hline Aspartate Transaminase (U/L) & $22(18-26)$ & $23(18-31)$ & 0.66 \\
\hline Alanine Transaminase (U/L) & $21(16-28)$ & $25(16-36)$ & 0.59 \\
\hline Right pleural effusion (mm) & $1.8(0-3.2)$ & $0(0-0)$ & $<0.001$ \\
\hline Left pleural effusion (mm) & $0.6(0-2.8)$ & $0(0-0.67)$ & $<0.001$ \\
\hline
\end{tabular}

${ }^{1}$ normally distributed parameters and pericardial effusion reported as mean \pm standard deviation; non-normally distributed parameters reported as median (interquartile range); categorical parameters reported as number (\%).

\subsection{Interobserver and Intraobserver Variabilities}

There was excellent agreement among the three observers for measurement of pericardial effusion on MRI (ICC $=0.939$ ). Intraobserver variability for measuring pericardial effusion on MRI was also excellent $(\mathrm{ICC}=0.997)$.

\subsection{Prevalence of Pericardial Effusion}

Pericardial effusion thickness was larger in ADPKD subjects compared to their matched controls for 59 pairs, smaller for 28 pairs and the same for 30 pairs (Mann-Whitney $p=0.001)$. Pericardial effusion $>5 \mathrm{~mm}$ was more prevalent in ADPKD subjects compared to controls (24 of $117(21 \%)$ vs. 4 of $117(3 \%), p<0.001)$ (Table 2). Pericardial effusion $>10 \mathrm{~mm}$ showed a strong trend toward greater prevalence in ADPKD subjects compared to controls (5 of $117(4 \%)$ vs. 0 of 117 controls $(0 \%), p=0.06)$. Comparisons of clinical, laboratory and genotype parameters between ADPKD patients with pericardial effusion $\leq 5 \mathrm{~mm}$ versus $>5 \mathrm{~mm}$ are shown in Table 3 .

Table 3. Demographic data in ADPKD subjects (pericardial effusion $\leq 5 \mathrm{~mm}$ vs. pericardial effusion $>5 \mathrm{~mm}$ ).

\begin{tabular}{lccc}
\hline \multicolumn{1}{c}{$\begin{array}{c}\text { Pericardial Effusion: } \\
\text { Characteristic }\end{array}$} & $\begin{array}{c}\leq \mathbf{5} \mathbf{~ m m} \\
\boldsymbol{n}=\mathbf{9 3}\end{array}$ & $\begin{array}{c}\mathbf{> 5} \mathbf{m m} \\
\boldsymbol{n}=\mathbf{2 4}\end{array}$ & $\boldsymbol{p}$ Value \\
\hline Age & $49 \pm 13$ & $42 \pm 11$ & 0.01 \\
\hline Male: female & $43: 50$ & $7: 17$ & 0.32 \\
\hline $\begin{array}{l}\text { White:Black:Asian:mixed:Native } \\
\text { American:unknown }\end{array}$ & $56: 7: 5: 4: 1: 13$ & $14: 2: 0: 2: 0: 3$ & - \\
\hline Body Mass Index $\left(\mathrm{kg} / \mathrm{m}^{2}\right)$ & $25(23-28)$ & $25(23-30)$ & 0.69 \\
\hline
\end{tabular}


Table 3. Cont.

\begin{tabular}{|c|c|c|c|}
\hline $\begin{array}{l}\text { Pericardial Effusion: } \\
\text { Characteristic }\end{array}$ & $\begin{array}{l}\leq 5 \mathrm{~mm} \\
n=93\end{array}$ & $\begin{array}{l}>5 \mathrm{~mm} \\
n=24\end{array}$ & $p$ Value \\
\hline Systolic Blood Pressure (mmHg) & $129 \pm 14$ & $125 \pm 25$ & 0.32 \\
\hline $\begin{array}{l}\text { Estimated Glomerular Filtration Rate } \\
\left(\mathrm{mL} / \mathrm{min} / 1.73 \mathrm{~m}^{2}\right)\end{array}$ & $65(45-89)$ & $68(46-93)$ & 0.72 \\
\hline Right pleural effusion (mm) & $3.3(2.1-4.6)$ & $0.7(0-2.5)$ & $<0.001$ \\
\hline Left pleural effusion $(\mathrm{mm})$ & $2.4(0.4-4)$ & $0(0-2.6)$ & 0.004 \\
\hline Tolvaptan use & $14(15 \%)$ & $4(17 \%)$ & 0.85 \\
\hline Increased fluid intake (>3 L/day) & $35(38 \%)$ & $8(33 \%)$ & 0.70 \\
\hline Thyroid stimulating hormone (mIU/mL) & $1.7(1.2-2.3)$ & $1.6(1.1-2.3)$ & 0.58 \\
\hline Blood Urea Nitrogen (mg/dL) & $21(17-30)$ & $19(15-25)$ & 0.26 \\
\hline Albumin (g/dL) & $4.2(4.1-4.4)$ & $4.1(3.9-4.3)$ & 0.18 \\
\hline Aspartate Transaminase (U/L) & $21(19-26)$ & $22(18-26)$ & 0.43 \\
\hline Alanine Transaminase (U/L) & $19(16-30)$ & $21(15-28)$ & 0.78 \\
\hline Total Kidney Volume/height & $994(660-1305)$ & $803(519-1470)$ & 0.41 \\
\hline Liver volume (mL) & $1895(1523-2321)$ & $1792(1517-2125)$ & 0.79 \\
\hline Spleen volume (mL) & $246(208-309)$ & $234(187-285)$ & 0.24 \\
\hline Cisterna chyli diameter (mm) & $4(3-5)$ & $4(3-5)$ & 0.84 \\
\hline Genotype data available (\%) & $59(63 \%)$ & $16(67 \%)$ & 0.09 \\
\hline PKD1 ${ }^{1}$-only Mutation (\%) & 29 of $59(49 \%)$ & 11 of $16(69 \%)$ & 0.16 \\
\hline Truncating $^{2}$ & 20 of $29(69 \%)$ & 4 of $11(36 \%)$ & 0.07 \\
\hline Nontruncating $^{2}$ & 6 of $29(21 \%)$ & 5 of $11(45 \%)$ & 0.07 \\
\hline PKD2 ${ }^{3}$-only Mutation (\%) & 13 of $59(22 \%)$ & 1 of $16(6 \%)$ & 0.15 \\
\hline Truncating & 13 of $13(100 \%)$ & 1 of $1(100 \%)$ & 1 \\
\hline Nontruncating & 0 of 13 & 0 of 1 & 1 \\
\hline PKD1 \& PKD2 Mutation (\%) & 1 of $59(2 \%)$ & 1 of $16(6 \%)$ & 0.32 \\
\hline No mutation detected (\%) & 16 of $59(27 \%)$ & 3 of $16(19 \%)$ & 0.49 \\
\hline
\end{tabular}

${ }^{1}$ PKD1, polycystic kidney disease $1 ;{ }^{2}$ data on truncation was not available for 5 subjects with PKD1 genotyped at other institutions, ${ }^{3}$ PKD2, polycystic kidney disease 2.

Although there were four ADPKD subjects with rheumatologic disease, none of these subjects had pericardial effusion thickness $>5 \mathrm{~mm}$. Furthermore, none of the ADPKD subjects with pericardial effusion thickness $>5 \mathrm{~mm}$ had clinical evidence of heart failure. Increased fluid intake in ADPKD subjects did not correlate with pericardial fluid thickness, as shown in Tables 3 and 4. One ADPKD subject had mildly elevated TSH, $6.6 \mathrm{mIU} / \mathrm{mL}$, however this patient had only minimal pericardial fluid with a thickness of $1 \mathrm{~mm}$.

Table 4. Bivariate correlations between pericardial effusion thickness and clinical/laboratory variables in ADPKD subjects.

\begin{tabular}{ccc}
\hline Variables & Correlation Coefficient & $p$ Value \\
\hline Age & -0.28 & 0.003 \\
\hline Gender & 0.24 & 0.010 \\
\hline Body Mass Index & 0.01 & 0.892 \\
\hline Systolic Blood Pressure & -0.15 & 0.118 \\
\hline
\end{tabular}


Table 4. Cont.

\begin{tabular}{ccc}
\hline Variables & Correlation Coefficient & $p$ Value \\
\hline Right pleural effusion & 0.50 & 0.00000001 \\
\hline Left pleural effusion & 0.37 & 0.00004 \\
\hline Total Kidney Volume/height & 0.06 & 0.498 \\
\hline Liver volume & 0.06 & 0.557 \\
\hline Spleen volume & 0.13 & 0.164 \\
\hline Cisterna chyli diameter & 0.03 & 0.787 \\
\hline Serum Creatinine & 0.07 & 0.460 \\
\hline Blood Urea Nitrogen & 0.03 & 0.748 \\
\hline Estimated Glomerular Filtration Rate & -0.04 & 0.651 \\
\hline Albumin & -0.003 & 0.970 \\
\hline Aspartate Transaminase & -0.17 & 0.066 \\
\hline Alanine Transaminase & -0.06 & 0.497 \\
\hline Thyroid stimulating hormone & -0.06 & 0.54 \\
\hline Tolvaptan use & -0.03 & 0.75 \\
\hline Increased fluid intake & -0.14 & 0.12 \\
\hline
\end{tabular}

\subsection{Correlation with Laboratory and Imaging Parameters}

Upon bivariate linear regression analysis, Table 4, pericardial effusion thickness on MRI of ADPKD subjects significantly correlated with gender, right and left pleural effusion, and negatively correlated with age. There was no correlation with BMI, blood pressure, liver volume, kidney volume, spleen volume, cisterna chyli diameter, AST, ALT, albumin, BUN and eGFR. Multivariate regression analysis (Table 5) including age, gender, and right pleural effusion, showed that female gender $(p=0.03)$ and right pleural effusion $(p=0.0000003)$ were associated with the presence of pericardial effusion $(p<0.0001)$.

Table 5. Multivariate analysis including variables with $p$ value $<0.05$ upon bivariate analysis on ADPKD subjects.

\begin{tabular}{cccccccc}
\hline & Coefficient & Standard Error & t Stat & $p$-Value & Lower 95\% & Upper 95\% \\
\hline Intercept & 2.24 & 1.25 & 1.79 & 0.08 & -0.23 & 4.72 \\
\hline Pleural effusion & 0.60 & 0.11 & 5.47 & 0.0000003 & 0.38 & 0.81 \\
\hline Age & -0.03 & 0.02 & -1.17 & 0.24 & -0.07 & 0.02 \\
\hline Gender & 1.21 & 0.54 & 2.24 & 0.03 & 0.14 & 2.28 \\
\hline
\end{tabular}

\subsection{Comparing MRI and Echocardiography Measures of Pericardial Effusion}

For the 46 ADPKD subjects in whom protocol echocardiography was also available within 1 year of the MRI, on average, the retrospective measurement of pericardial effusion thickness was similar in MRI and echocardiography. In these 46 subjects, there were eight with pericardial effusions $>5 \mathrm{~mm}$ on MRI. All eight had normal left ventricular ejection fraction. For the three ADPKD subject in whom echo showed a left ventricular ejection fraction $<55 \%$, pericardial effusion thickness measured 0,1 and $1 \mathrm{~mm}$. Although pericardial fluid was found retrospectively on the echocardiography matching the MRI findings in seven subjects, pericardial fluid was prospectively reported in only two of those seven cases. The echocardiography report described a small pericardial effusion in a patient with $6 \mathrm{~mm}$ pericardial effusion on MRI and echocardiography described trace pericardial 
fluid in a patient with a $12 \mathrm{~mm}$ pericardial effusion on MRI. In the other five of the seven subjects, echocardiography reported "no pericardial effusion", although MRI showed pericardial effusions measuring 6,6,7,7 and $8 \mathrm{~mm}$. However, on retrospective review of the echocardiography, pericardial effusions were identified, but asymmetrically distributed more around the right ventricle rather than circumferentially (Figure 3). A summary of other echo findings are provided in Supplemental Table S4; we found no evidence of any difference between ADPKD and control groups in measures of cardiac structure or function.

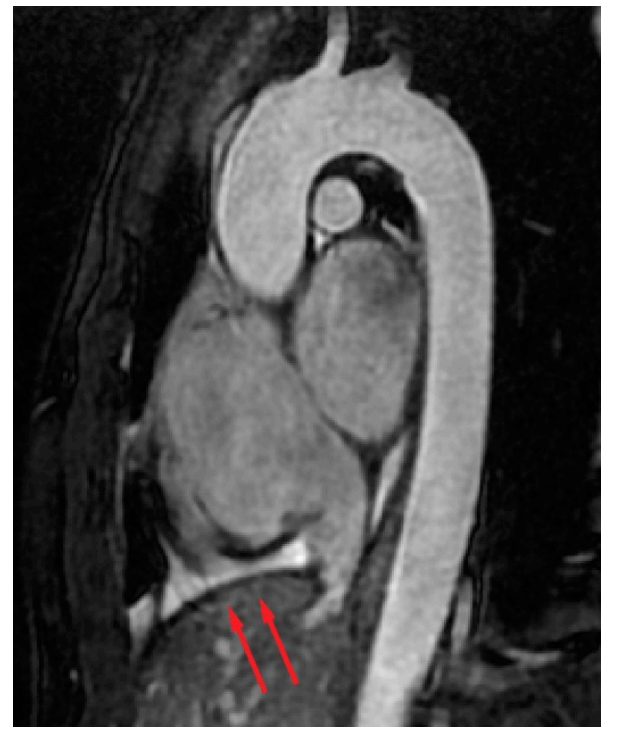

(A)

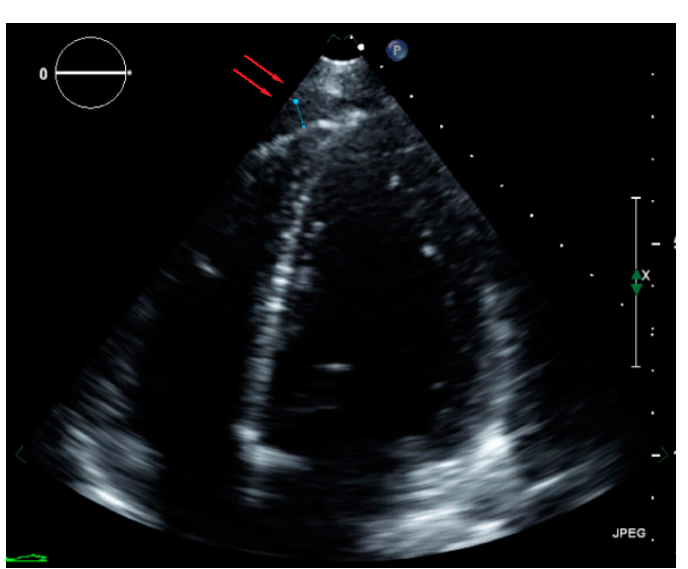

(B)

Figure 3. Asymmetrically distributed pericardial effusion (red arrows) on (A) MRI and (B) echocardiography.

\subsection{Genotype}

Among 75 ADPKD subjects with genotype data available, 56 were found to have pathogenic mutation of either PKD1 or PKD2-there were 40 subjects with PKD1-only mutation, 14 subjects with PKD2-only mutation and 2 subjects with both PKD1 and PKD2 mutations. Nineteen subjects did not have a pathogenic PKD1 or PKD2 mutation identified despite a clinical diagnosis of ADPKD based upon Pei criteria. Furthermore, 24 of the 40 $(60 \%)$ PKD1 mutations were truncating and all of the PDK2 mutations were truncating. No statistically significant correlation between genotype and pericardial effusion was detected. Among two subjects who had both PKD1 and PKD2 mutations, one had pericardial effusion $>5 \mathrm{~mm}$.

\subsection{Clinical Effects of Pericardial Effusion}

No subject had a hemodynamically significant pericardial effusion or symptoms attributed to their pericardial effusion. Moreover, no subject required pericardiocentesis or other procedure related to the pericardial effusion.

\section{Discussion}

This retrospective cross-sectional study analyzing ECG-gated cine MRI on 117 ADPKD subjects comparing to age, gender, eGFR matched controls demonstrates that pericardial effusion occurs commonly in ADPKD with a prevalence of $21 \%$ compared to $3 \%$ in the control population. Multivariate analysis found that female gender and concurrent pleural effusion are associated with the presence of pericardial effusion in ADPKD. Pericardial effusion was observed in subjects with PKD1 and PKD2 mutations.

When comparing echocardiography to ECG-gated MRI, most (seven of eight) pericardial effusions $>5 \mathrm{~mm}$ on MRI were retrospectively detected on echocardiography when 
the reader was aware of MRI results. However, only two of eight effusions seen on MRI were reported on the echocardiography initial reading performed blinded to MRI results. This echocardiography oversight may be due to the location of the effusions around the right ventricle, which is readily evaluated by MRI but not as easily evaluated by echocardiography due to the sternum and ribs limiting acoustic windows. Moreover, pericardial effusion has been only rarely reported in ADPKD subjects [13-15]. Qian et al. reported pericardial effusion in ADPKD on CT scans [14]. However, CT is less sensitive and less convincing than MRI for detection of effusions [16,17]. Furthermore, ECG-gated cardiac $\mathrm{CT}$ is rarely performed on subjects with ADPKD.

Multivariate analysis found pericardial effusion to be associated with female gender and pleural effusion. The association of pleural and pericardial effusions suggests the possibilities of salt/water overload, heart failure with preserved ejection fraction, serositis or lymphatic problems. Our limited data on fluid intake by ADPKD subjects was not correlated with pericardial effusion thickness. We found no evidence of heart failure or rheumatologic disease in the ADPKD subjects with pericardial effusions $>5 \mathrm{~mm}$. However, the previous finding of an enlarged cisterna chyli in ADPKD subjects [18] raises the possibility of high fluid throughput occurring in the thorax in ADPKD, possibly reflecting increased flow through the lymphatic circulation. Comparison to cisterna chyli diameter in the control subjects was not possible in this study because the cardiac MRI did not include the cisterna chyla within the field of view of T2 weighted images. The absence of a significant association with standard ADPKD imaging biomarkers, including kidney and liver volumes $[19,20]$, suggests pericardial effusion may be an independent ADPKD biomarker corresponding to a different aspect of the disease compared to the other standard imaging and laboratory ADPKD biomarkers that are more focused on other organs, e.g., kidney and liver.

The clinical significance of pericardial effusion in ADPKD patients is not clear. No subject in this study had hemodynamically significant pericardial effusion and none required pericardiocentesis or other therapeutic procedures. However, hemodynamically significant pericardial effusion in an ADPKD patient has been reported [15]. Therefore, follow-up pericardial imaging to assess the progression of an effusion would be reasonable to determine stability. In addition, evaluation of other potential etiologies of a pericardial effusion, (e.g., hypothyroidism, connective tissue diseases), should be considered where clinically indicated. Since the etiology and prognostic value of pericardial effusion are unknown in ADPKD, future research is warranted.

The strengths of this study include a relatively large sample size for this condition, the non-ADPKD control group and prospective analysis of MRI and other demographic/laboratory data from a well-characterized ADPKD population within the Rogosin ADPKD Repository. Limitations of this study include the small number of subjects who underwent both echocardiography and MRI as well as the time interval between those studies, thereby obscuring a comparison of these diagnostic tests for assessing pericardial effusion. The number of subjects evaluated by PKD gene testing was also relatively small, thus, conclusions regarding the possible association of PKD mutation [21] locus on the prevalence of pericardial effusion were not feasible. Further investigation of the relationship of pericardial effusion to genotype in a larger ADPKD cohort is needed. Retrospective analysis of existing research cohort data may have created observer biases and there may have been incomplete medical information for ADPKD and control subjects. Tolvaptan is a type 2 vasopressin receptor antagonist that is approved for the treatment of ADPKD. This aquaretic drug can cause significant dehydration, and consequently, may have attenuated pericardial effusion thickness underestimating the prevalence of pericardial effusion in those ADPKD subjects on tolvaptan.

\section{Conclusions}

This retrospective cross-sectional study showed a relatively high prevalence of pericardial effusion in ADPKD as well as the association of pericardial effusion with female 
gender and the presence of pleural effusion in ADPKD. The etiology and clinical significance of pericardial effusion in ADPKD is unknown but its association with pleural effusion may have implication regarding the pathophysiology of ADPKD which warrants further investigation.

Supplementary Materials: The following supporting information can be downloaded at: https: / / www.mdpi.com/article/10.3390/jcm11041127/s1, Table S1: Primary indications for Cardiac MRI in control population; Table S2: Medications to control blood pressure in ADPKD and control subjects (note that some subjects are on no blood pressure medications and some are on more than 1 medication so it does not add up to 117 in each column); Table S3: Causes of chronic kidney disease in 44 control subjects with eGFR $<60 \mathrm{~mL} / \mathrm{min} / 1.73 \mathrm{~m}^{2}$; Table S4: Cardiac findings in ADPKD subjects with echo within 1 year of MRI and corresponding controls, mean \pm standard deviation (min-max).

Author Contributions: Conceptualization, M.R.P., J.B. and J.L.; methodology, M.R.P. and J.L.; formal analysis of images, M.R.P., K.F. and J.L.; data curation, all authors; writing—original draft preparation, J.L.; writing—review and editing, all authors; supervision, M.R.P. and J.B.; project administration, J.B. and M.R.P. All authors have read and agreed to the published version of the manuscript.

Funding: Research reported in this publication was supported by the National Center for Advancing Translational Science of the National Institute of Health under award number UL1TR002384 and the Shaw Foundation.

Institutional Review Board Statement: The study was conducted according to the guidelines of the Declaration of Helsinki, and approved by the Institutional Review Board of Weill Cornell Medicine (protocol codes 0304006105 and 1610017623 approved 7/7/2017).

Informed Consent Statement: Informed consent was obtained from all subjects enrolled in Rogosin Institute PKD repository. For patients not in the PKD repository, the requirement of consent to review existing medical images and data was waived because of minimal risk.

Data Availability Statement: The data presented in this study are available on request from the corresponding author subject to IRB approval. The data are not publicly available due to HIPPA regulations.

Conflicts of Interest: The authors declare no conflict of interest.

\section{References}

1. Cornec-Le Gall, E.; Alam, A.; Perrone, R.D. Autosomal dominant polycystic kidney disease. Lancet 2019, 393, 919-935. [CrossRef]

2. Perumareddi, P.; Trelka, D.P. Autosomal Dominant Polycystic Kidney Disease. Prim. Care 2020, 47, 673-689. [CrossRef]

3. Irazabal, M.V.; Rangel, L.J.; Bergstralh, E.J.; Osborn, S.L.; Harmon, A.J.; Sundsbak, J.L.; Bae, K.T.; Chapman, A.B.; Grantham, J.J.; Mrug, M.; et al. Imaging classification of autosomal dominant polycystic kidney disease: A simple model for selecting patients for clinical trials. J. Am. Soc. Nephrol. 2015, 26, 160-172. [CrossRef]

4. Krishnappa, V.; Vinod, P.; Deverakonda, D.; Raina, R. Autosomal dominant polycystic kidney disease and the heart and brain. Cleve Clin. J. Med. 2017, 84, 471-481. [CrossRef]

5. Perrone, R.D.; Malek, A.M.; Watnick, T. Vascular complications in autosomal dominant polycystic kidney disease. Nat. Rev. Nephrol. 2015, 11, 589-598. [CrossRef]

6. Vlak, M.H.; Algra, A.; Brandenburg, R.; Rinkel, G.J. Prevalence of unruptured intracranial aneurysms, with emphasis on sex, age, comorbidity, country, and time period: A systematic review and meta-analysis. Lancet Neurol. 2011, 10, 626-636. [CrossRef]

7. Chebib, F.T.; Hogan, M.C.; El-Zoghby, Z.M.; Irazabal, M.V.; Senum, S.R.; Heyer, C.M.; Madsen, C.D.; Cornec-Le Gall, E.; Behfar, A.; Harris, P.C.; et al. Autosomal Dominant Polycystic Kidney Patients May Be Predisposed to Various Cardiomyopathies. Kidney Int. Rep. 2017, 2, 913-923. [CrossRef]

8. Lumiaho, A.; Ikäheimo, R.; Miettinen, R.; Niemitukia, L.; Laitinen, T.; Rantala, A.; Lampainen, E.; Laakso, M.; Hartikainen, J. Mitral valve prolapse and mitral regurgitation are common in patients with polycystic kidney disease type 1. Am. J. Kidney Dis. 2001, 38, 1208-1216. [CrossRef]

9. Pei, Y.; Obaji, J.; Dupuis, A.; Paterson, A.D.; Magistroni, R.; Dicks, E.; Parfrey, P.; Cramer, B.; Coto, E.; Torra, R.; et al. Unified criteria for ultrasonographic diagnosis of ADPKD. J. Am. Soc. Nephrol. 2009, 20, 205-212. [CrossRef]

10. Pei, Y.; Hwang, Y.H.; Conklin, J.; Sundsbak, J.L.; Heyer, C.M.; Chan, W.; Wang, K.; He, N.; Rattansingh, A.; Atri, M.; et al. Imaging-based diagnosis of autosomal dominant polycystic kidney disease. J. Am. Soc. Nephrol. 2015, 26, 746-753. [CrossRef]

11. Levey, A.S.; Stevens, L.A.; Schmid, C.H.; Zhang, Y.L.; Castro, A.F., 3rd; Feldman, H.I.; Kusek, J.W.; Eggers, P.; Van Lente, F.; Greene, T.; et al. A new equation to estimate glomerular filtration rate. Ann. Intern. Med. 2009, 150, 604-612. [CrossRef] 
12. Mitchell, C.; Rahko, P.S.; Blauwet, L.A.; Canaday, B.; Finstuen, J.A.; Foster, M.C.; Horton, K.; Ogunyankin, K.O.; Palma, R.A.; Velazquez, E.J. Guidelines for Performing a Comprehensive Transthoracic Echocardiographic Examination in Adults: Recommendations from the American Society of Echocardiography. J. Am. Soc. Echocardiogr. 2019, 32, 1-64. [CrossRef]

13. Fernandez, G.A.P.; Ismail, M.Y. Autosomal Dominant Polycystic Kidney Disease and Pericardial Effusion. Oman Med. J. 2018, 33, 429-432. [CrossRef]

14. Qian, Q.; Hartman, R.P.; King, B.F.; Torres, V.E. Increased occurrence of pericardial effusion in patients with autosomal dominant polycystic kidney disease. Clin. J. Am. Soc. Nephrol. 2007, 2, 1223-1227. [CrossRef]

15. Çelik, A.; Bezgin, T.; Çağdaş, M. Autosomal dominant polycystic kidney disease with liver involvement and left ventricular noncompaction: An unusual coexistence. Anatol. J. Cardiol. 2021, 25, 5020. [CrossRef]

16. Cosyns, B.; Plein, S.; Nihoyanopoulos, P.; Smiseth, O.; Achenbach, S.; Andrade, M.J.; Pepi, M.; Ristic, A.; Imazio, M.; Paelinck, B.; et al. European Association of Cardiovascular Imaging (EACVI) position paper: Multimodality imaging in pericardial disease. Eur. Heart J. Cardiovasc. Imaging 2015, 16, 12-31. [CrossRef]

17. Alter, P.; Figiel, J.H.; Rupp, T.P.; Bachmann, G.F.; Maisch, B.; Rominger, M.B. MR, CT, and PET imaging in pericardial disease. Heart Fail. Rev. 2013, 18, 289-306. [CrossRef]

18. Thimmappa, N.D.; Blumenfeld, J.D.; Cerilles, M.A.; Dunning, A.; Donahue, S.L.; Bobb, W.O.; Zhang, H.L.; Prince, M.R. Cisterna chyli in autosomal dominant polycystic kidney disease. J. Magn. Reason. Imaging 2015, 41, 142-148. [CrossRef]

19. Tangri, N.; Hougen, I.; Alam, A.; Perrone, R.; McFarlane, P.; Pei, Y. Total Kidney Volume as a Biomarker of Disease Progression in Autosomal Dominant Polycystic Kidney Disease. Can. J. Kidney Health Dis. 2017, 4, 1-6. [CrossRef]

20. Neijenhuis, M.K.; Kievit, W.; Perrone, R.D.; Sloan, J.A.; Erwin, P.; Murad, M.H.; Gevers, T.J.G.; Hogan, M.C.; Drenth, J.P.H. The effect of disease severity markers on quality of life in autosomal dominant polycystic kidney disease: A systematic review, meta-analysis and meta-regression. BMC Nephrol. 2017, 18, 169. [CrossRef]

21. Bitarafan, F.; Garshasbi, M. Molecular genetic analysis of polycystic kidney disease 1 and polycystic kidney disease 2 mutations in pedigrees with autosomal dominant polycystic kidney disease. J. Res. Med. Sci. 2019, 24, 44. [CrossRef] [PubMed] 